\title{
Molecular dynamics simulations of ultrathin water film confined between flat diamond plates
}

\author{
A.V.Khomenko, N.V.Prodanov \\ Sumy State University, 2 Rimskiy-Korsakov Str., 40007 Sumy, Ukraine
}

Received May 7, 2008, in final form September 30, 2008

\begin{abstract}
Molecular dynamics simulations of ultrathin water film confined between atomically flat rigid diamond plates are described. Films with thickness of one and two molecular diameters are concerned and TIP4P model is used for water molecules. Dynamical and equilibrium characteristics of the system for different values of the external load and shear force are investigated. An increase of the external load causes the transition of the film to a solidlike state. This is manifested in a decrease of the diffusion constant and in the ordering of the liquid molecules into quasidiscrete layers. For two-layer film under high loads, the molecules also become ordered parallel to the surfaces. Time dependencies of the friction force and the changes of its average value with the load are obtained. In general, the behaviour of the studied model is consistent with the experimental results obtained for simple liquids with spherical molecules.
\end{abstract}

Key words: boundary lubrication, molecular dynamics, ultrathin liquid film

PACS: 61.20.Ja, 61.20.Ne, 61.30.Hn, 68.35.Af

\section{Introduction}

The study of friction at the atomic level appears to be important both from fundamental and practical viewpoints. The development of nanotechnologies and the creation of new devices on their basis require a deep understanding of nanoscale processes leading to wear and friction [1-4]. In nanotribological studies there is a phenomenon which has a special place and is called boundary lubrication. It corresponds to the situation when the rubbing surfaces are separated by an ultrathin (a few molecular diameters) lubricant film. Boundary lubrication is important not only for nanotechnology. This is observed under ordinary conditions, because nearly always there is a lubricant between the solids (called "the third bodies" by tribologists) - either a specially chosen lubricant film, or wear debris produced by sliding, or water adsorbed from air, etc. Boundary lubrication is also important in macromachines at stop/start moments, when the lubricant is squeezed out of the contact area and the surfaces come into direct contact [1-4].

As the experiments with surface-force apparatus (SFA) and the computer simulations show, the behaviour of ultrathin liquid film confined between two solid substrates entirely differs from the bulk one. The main features of a boundary lubricant are as follows $[1,4-6]$ :

1. Liquid molecules confined between two atomically flat surfaces become more ordered and tend to organize quasidiscrete layers, where the average local density of the liquid oscillates with the distance normal to the boundaries.

2. The mobility of molecules in confined films considerably decreases in comparison with bulk liquids. This is manifested in a decrease of the diffusion constant and in an increase of the viscosity and molecular relaxation times. The "effective" viscosity can be $10^{5}$ times larger and relaxation times can be $10^{10}$ times slower than the bulk value.

3. Boundary lubricant may exhibit two different responses in shear: a liquidlike, in which the liquid responds to the deformation by flow, and a solidlike characterized by observation of the development of yield stress in the confined fluid. In computer simulations with simple Lennard-Jones (LJ) liquids between atomically structured surfaces the existence of abrupt 
liquid to solid transitions in films thinner than 6 molecular diameters was predicted, with the molecules becoming ordered both perpendicular and parallel to the surfaces. However, the horizontal order disappears for unstructured surfaces. In confined fluids both positional and orientational orderings are caused not only by mutual interactions of liquid molecules but also by the presence of two solid surfaces close together.

4. For velocities of moving substrate less than some critical value, the stick-slip motion may be observed. The stick-slip regime is characterized by intermittent stops (stick) and slips.

Analytical models are widely used in explaining the experimental results. However, these models are applicable only in special cases and, as a rule, they are only qualitative [7-10]. Computer simulations offer an additional tool for studying the atomic-scale friction and wear. In particular, molecular dynamics (MD) simulations are very helpful in understanding the nanotribological processes $[6,11-14]$.

In this article the MD simulations of ultrathin water film confined between atomically flat diamond plates are described. The choice of water as a liquid for modelling is motivated by its importance, ubiquity and unique tribological properties $[5,15]$. The introduction of a water monolayer (only $0.25 \mathrm{~nm}$ thick) between atomically flat mica surfaces of the SFA causes a decrease of friction by more than an order of magnitude [16]. The effectiveness of a water monolayer in lowering the friction force is attributed to the "hydrophilicity" of the mica surface (mica is "wetted" by water) and to the existence of a strongly repulsive short-range hydration force between these surfaces in aqueous solutions, which effectively removes the adhesion-controlled contribution to the friction force. Under the described conditions the friction force obeys the first Amontons' law which states that frictional force is proportional to the external load.

The behaviour of the confined water molecules has been intensively explored using MD technique for the recent years [17-22]. Equilibrium structural and dynamical properties of several water molecule models in the confining pores of a variety of shapes have been dealt with in these works.

Simulations of TIP3P water molecules in the hydrophobic cylindrical cavity of different radii [17] and SPC/E molecules in a hydrophilic cylindrical Vycor glass pore $[18,19]$ reveal nonuniform distribution of water molecules which is manifested in the oscillating density profiles. In the first case, poorly defined concentric shells are formed, and the mobility of molecules defined by the diffusion constant considerably decreases with a decrease of the cavity's radius. In the second case, when water adsorbs, a double layer is formed and anomalous diffusion may be observed. In a similar way, MD simulations of SPC/E water molecules confined in silica gel [20] show the formation of a water shell around the hydrophilic silica particles. However, no secondary shell is observed either for high density or for low one, which is attributed to a high roughness of the silica surface.

MD simulations on the adsorption of SPC/E water in attractive and repulsive slit pores [21] also reveal the formation of layers without lateral ordering for both types of pores for high densities of molecules. The obtained results indicate that the type of interactions between water molecules and the surfaces can greatly effect the behaviour of the confined liquid. Similarly, modellings of SPC water confined in the interdomain region of a multidomain protein, namely BphC enzyme [22], suggest that local surface curvature and hydrophobicity have a significant effect on the water structure and dynamics. Thus, MD simulations of confined water under different conditions show that water reveals general features of confined liquids mentioned above.

The purpose of the present work is to investigate the behaviour of the water film consisting of TIP4P molecules confined between totally rigid flat diamond plates and to check the model for consistency with the experiments and other simulations. The present study concerns not only the equilibrium structural and dynamical properties of water, but also the tribological characteristics, which were not involved in the described models. The results in turn may give us estimates of the reliability of using the TIP4P model for water molecules, and the applicability of the approximation of absolutely rigid plates in the modelling of tribological systems. 


\section{Model}

In the simulations [17-22], models are built in such a way that they reflect the experimental investigations of water in porous media. That is why the confining walls are fixed and in some works the model consists both of a pore and a bulk region in order to establish equilibrium conditions between the bulk phase and the fluid in the pore [6,21]. However, in the present study the model is constructed to simulate the nanotribological experiments with SFA. Here, the radius of the contact area of the two atomically flat surfaces is of the order of micrometers, the distance between the plates can be changed with the normal load and the upper surface can be moved laterally (or sheared) [2]. When two walls slide past each other, the work is done on the system. The energy is ultimately converted into heat and should go away from the interface mainly to the substrates $[1,11]$ due to a relatively large contact area. Therefore, while simulating SFA experiments it is not appropriate to connect a small slit with the bulk. A more reasonable choice is the use of periodic boundary conditions in the horizontal plane and the heat flow is provided by coupling the particles to a heat bath. The described approach is used in a number of simulations [11-14] where a planar, Couette geometry is considered. A similar model is introduced in the present work.

A thin film of water molecules is confined between two solid walls with periodic boundary conditions in the plane of the walls. Each wall consists of 1152 atoms forming two (001) crystallographic planes of diamond lattice. Although the experimental study of friction of the diamond plates separated by an ultrathin water film has not been carried out yet, still the diamond is transparent for the light and therefore can be used in the SFA. The walls are considered to be absolutely rigid and the elasticity of plates is not included into the model. However, taking into account that diamond is one of the hardest materials we decided to verify the approximation. The water films of one and two molecular diameter thicknesses have been investigated. At the beginning of simulation one water layer consists of 196 molecules. The maximum value of particles involved in the simulations is 2696 .

Figure 1 shows the initial configurations of the studied system for both values of the film's thickness. The initial state of the film and of the crystalline plate (the view is from the top in the negative direction of the axis $z$ ) are shown in figure 2 .

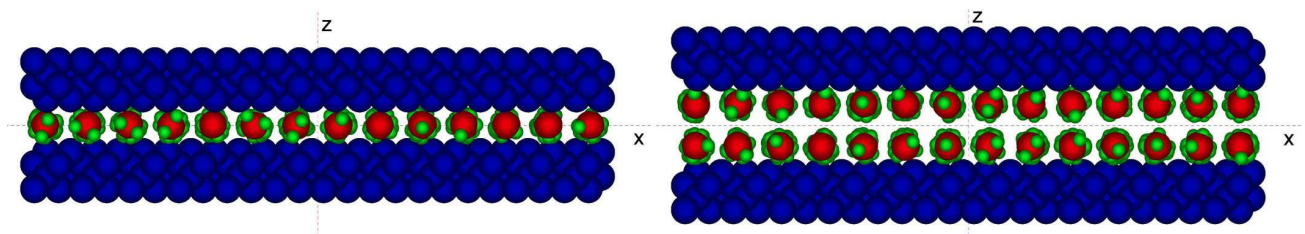

Figure 1. The initial configuration of the studied system with one (left) and two (right) layers of water molecules. Carbon, oxygen and hydrogen atoms are shown as blue, red and green balls, respectively.

The empiric values of the covalent radii of atoms are used for visualization. For carbon, oxygen and hydrogen they are 77,73 , and $37 \mathrm{pm}\left(1 \mathrm{pm}=10^{-12} \mathrm{~m}\right)$, respectively, the lattice constant

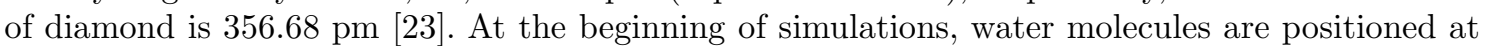
the sites of a simple cubic lattice with the lattice constant corresponding to the density value of $1048 \mathrm{~kg} \mathrm{~m}^{-3}$. Molecular orientation is randomly assigned, with each linear and angular velocity having a fixed magnitude defined by the temperature and a randomly chosen directions. The water film and the plates are incommensurate because the diamond lattice constant is not equal to the initial distance between water molecules. The initial distance (the gap) between the walls is $0.31 \mathrm{~nm}$ for one layer of water molecules and $0.62 \mathrm{~nm}$ for two layers. The horizontal dimensions of the computational cell are equal for $x$ and $y$ directions and their value is $42.81 \AA$.

In order to imitate the experiments, the modelling is performed for constant temperature and load applied to the plates. The constraint method is used to maintain a constant temperature value which is $298 \mathrm{~K}$. The external load is modeled by applying the constant force $L$ to each atom of the walls along the $z$ axis. The constant-force algorithm is used [14]. This means that shear is imitated 


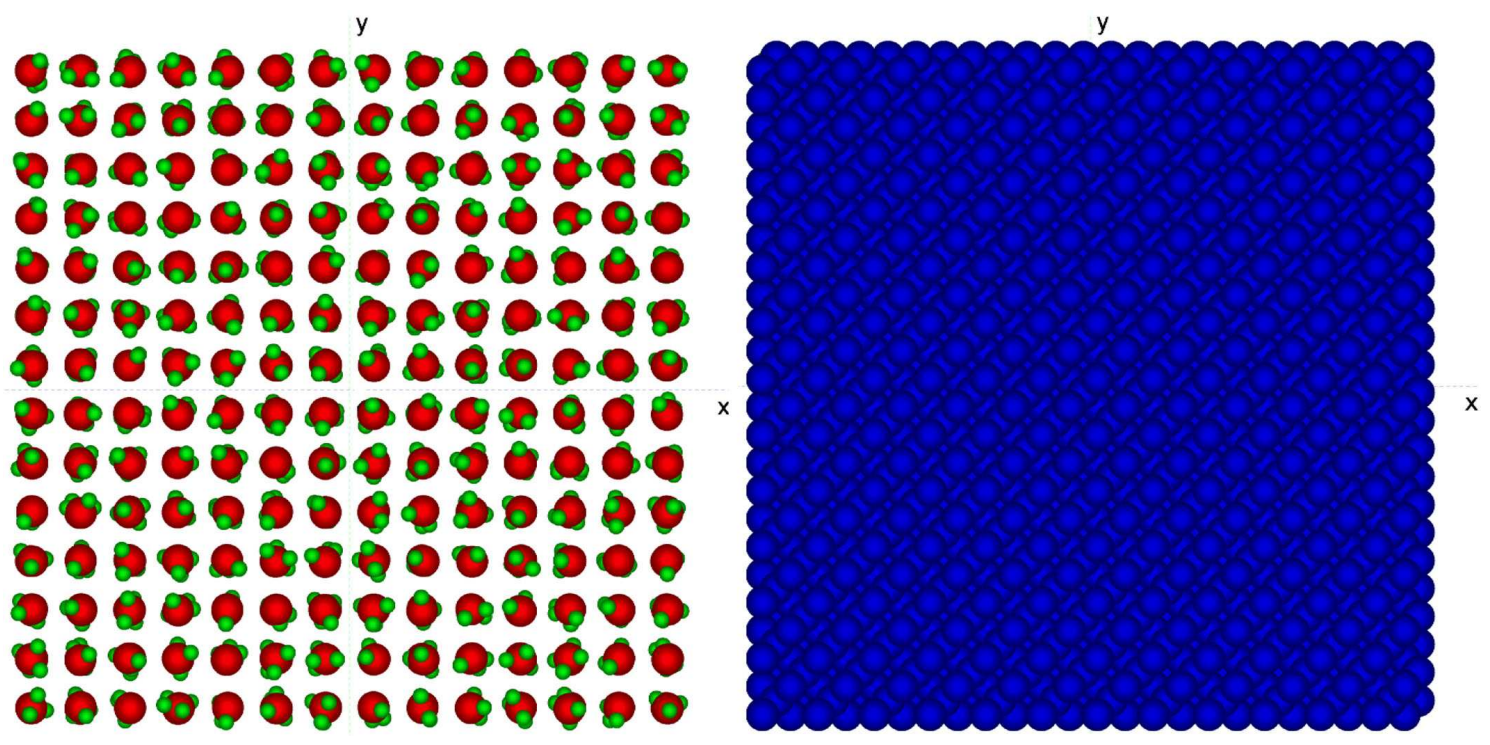

Figure 2. The arrangement of water molecules (left) and the form of diamond plate (right) in the $x y$ plane at the beginning of a simulation.

by applying constant horizontal shearing (i. e., driving) force $F_{\mathrm{S}}$ to each atom of the upper plate along the $x$ axis which corresponds to [010] direction.

The TIP4P model is used for water molecules [24]. It is based on four interaction sites located in planar configuration, two of which - labeled $\mathrm{M}$ and $\mathrm{O}$ - are associated with the oxygen nucleus, and two - labeled $\mathrm{H}$ - with protons. The interaction energy between two molecules $i$ and $j$ consists of a double sum over the interaction sites of both molecules; the terms in the sum, indexed by $k$ and $l$, allow for Coulomb interactions between the electric charges assigned to the sites, as well as contributions of LJ type,

$$
u_{i j}=\sum_{k \in i} \sum_{l \in j}\left(\frac{q_{k} q_{l}}{r_{k l}}+\frac{A_{k l}}{r_{k l}^{12}}-\frac{C_{k l}}{r_{k l}^{6}}\right)
$$

where $\mathbf{r}_{k l}=\mathbf{r}_{k}-\mathbf{r}_{l}$ is the distance between the sites $k$ and $l, r_{k l} \equiv\left|\mathbf{r}_{k l}\right|$. The corresponding force is

$$
\mathbf{f}_{i j}=\sum_{k \in i} \sum_{l \in j}\left(\frac{q_{k} q_{l}}{r_{k l}^{3}}+\frac{12 A_{k l}}{r_{k l}^{14}}-\frac{6 C_{k l}}{r_{k l}^{8}}\right) \mathbf{r}_{k l} .
$$

The charges appearing in the potential function are $q_{\mathrm{H}}=0.52 e, q_{\mathrm{O}}=0, q_{\mathrm{M}}=-2 q_{\mathrm{H}}$, where $e=1.6 \times 10^{-19} \mathrm{C}$. The parameters in the LJ part of the potential, which acts only between $\mathrm{O}$ sites, are $A_{\mathrm{OO}} \equiv A=600 \times 10^{3}(\mathrm{kcal} / \mathrm{mole}) \AA^{12}, C_{\mathrm{OO}} \equiv C=610(\mathrm{kcal} / \mathrm{mole}) \AA^{6}$, and $1 \mathrm{kcal} / \mathrm{mole}=$ $4184 \mathrm{~J} / \mathrm{mole}$.

Dimensionless units are used in the simulations. The length unit is $\sigma=(A / C)^{1 / 6}=3.154 \AA$, the energy unit is $\varepsilon=A /(4 \sigma)^{12}=0.155 \mathrm{kcal} / \mathrm{mole}$, the mass unit is the mass of a water molecule, $m=2.987 \times 10^{-27} \mathrm{~g}$ and the time unit is $t_{0}=\sqrt{m \sigma^{2} / \varepsilon}=5.253 \times 10^{-12} \mathrm{~s}$. Also in dimensionless units $q_{\mathrm{H}}=1$. The time step used is $\Delta t=0.0005$; in real units this equals to $2.627 \times 10^{-15} \mathrm{~s}$.

For the water-diamond interactions, only the interactions of $\mathrm{O}$ site of the water molecule with carbon atoms are assumed. The interaction potential is of LJ type

$$
u_{i j}= \begin{cases}4 \varepsilon_{\mathrm{CO}}\left[\left(\frac{\sigma_{\mathrm{CO}}}{r_{i j}}\right)^{12}-\left(\frac{\sigma_{\mathrm{CO}}}{r_{i j}}\right)^{6}\right], & r_{i j}<r_{\mathrm{c}}, \\ 0, & r_{i j} \geqslant r_{\mathrm{c}},\end{cases}
$$

where $r_{\mathrm{c}}=7.5 \AA$ (or 2.38 in dimensionless units) is the interaction cutoff distance, and $\varepsilon_{\mathrm{CO}}=2$, $\sigma_{\mathrm{CO}}=0.86$. The values of the latter parameters are chosen to fit the experimental fact that 
diamond is hydrophilic [25]. They correspond to the situation when the attractive forces between water molecules and carbon atoms have a twice greater magnitude than intermolecular water forces. The interaction between atoms of different plates is not introduced into the model.

The classical equations of motion are used in simulations. Due to the rigidity of diamond plates, the motion of their centers of masses is taken into consideration. The equations of motion for the upper plate are

$$
M \ddot{X}=F_{\mathrm{x}}+F_{\mathrm{S}} N_{\mathrm{p}}, \quad M \ddot{Y}=F_{\mathrm{y}}, \quad M \ddot{Z}=F_{\mathrm{z}}+L N_{\mathrm{p}},
$$

where $X, Y, Z$ are the coordinates of the center of mass of the upper plate, $N_{\mathrm{p}}$ is the number of atoms of the wall, $M=N_{\mathrm{p}} m_{\mathrm{C}}$ is the plate's mass, $m_{\mathrm{C}}=0.67$ is the carbon atomic mass, $F_{\mathrm{x}}, F_{\mathrm{y}}, F_{\mathrm{z}}$ are the components of the total force acting on the wall from the water. They are defined in a standard manner as the sum of the appropriate coordinate derivatives of the potential (3) with the negative sign for all molecules. Equations of motion for the lower plate are similar to (4). The difference is in the absence of the component responsible for shear like it is in the first equation in (4).

The equations of the translational motion (without a component responsible for the constancy of temperature) for the center of mass of the $i$ th molecule are as follows:

$$
\ddot{\mathbf{r}}_{i}=\sum_{j} \mathbf{F}_{i j}+\sum_{k} \mathbf{f}_{i k}
$$

where $\sum_{j} \mathbf{F}_{i j}$ is the force acting from all carbon atoms that are not further than $r_{\mathrm{c}}$ from the $i$ th molecule and it is defined in the way similar to the force for the surfaces; $\sum_{k} \mathbf{f}_{i k}$ is the force acting on the $i$ th molecule from other water molecules within the cutoff distance, the force is defined by equation (2). The rotational motion of water molecules is taken into account in the model and the equations of the rotational motion are expressed in terms of Hamilton's quaternions [24].

\section{Measurements, results and discussion}

Molecular dynamics simulations are performed for values of the load $L$ per atom in the range from 2 (which is equal to $6.838 \mathrm{pN}$ in real units) to $50(0.171 \mathrm{nN})$. The corresponding pressure acting on the plates varies from 0.43 to $10.751 \mathrm{GPa}$. The horizontal shearing force $F_{\mathrm{S}}$ acting on each atom of the upper plate has values from 0.5 (or $1.71 \mathrm{pN})$ to $10(34.3 \mathrm{pN}$ ) per atom and the resulting shear force acting on the plate changes from 1.97 to $394 \mathrm{nN}$. The maximum duration of the simulation is 52000 time steps or 136.6 ps. At each simulation during the first 2000 time steps the system is equilibrated and then the measurements are carried out. In spite of a rather short equilibration period used in the simulations it is sufficient for complete system relaxation as the calculation of the velocity distribution shows. There are two groups of simulations. The first group is characterized by the absence of shearing force and the measurement of the diffusion constant is performed. In the second group the shear of the upper plate takes place and the friction force is measured. The latter is presented by the first component in the right hand side of the first equation in (4).

The diffusion constant $D$ is calculated in two ways. In the first case the Einstein expression is used $[24]$

$$
D=\lim _{t \rightarrow \infty} \frac{1}{6 N_{\mathrm{m}} t}\left\langle\sum_{j=1}^{N_{\mathrm{m}}}\left[\mathbf{r}_{j}(t)-\mathbf{r}_{j}(0)\right]^{2}\right\rangle,
$$

where $N_{\mathrm{m}}$ is the number of molecules, $t$ is the time of measurements and $\mathbf{r}_{j}$ is the radius vector of the $j$ th molecule center of mass. In (6) angled brackets denote the average over a sufficiently large number of independent samples. Note, that under such severe confinements as in the considered problem the Einstein relation (6) is not accurate and gives only rough estimates of the diffusion constant [22]. Nevertheless, it still reflects the general behaviour of the mobility of molecules. 
The second way of calculating the diffusion constant is using the alternative Green-Kubo expression for $D$ based on the integrated velocity autocorrelation function $\varphi(t)[24,26]$

$$
\begin{gathered}
D=\frac{1}{3 N_{\mathrm{m}}} \int_{0}^{\infty} \varphi(t) \mathrm{d} t, \\
\varphi(t)=\left\langle\sum_{j=1}^{N_{\mathrm{m}}} \boldsymbol{v}_{j}(t) \cdot \boldsymbol{v}_{j}(0)\right\rangle,
\end{gathered}
$$

where $\boldsymbol{v}_{j}$ is the velocity of the $j$ th molecule.

Figure 3 shows the calculated time dependencies of the velocity autocorrelation function of water molecules for different loads. It is apparent that with the increase of the load, the number and frequency of oscillations increase. This indicates that molecule velocities become more correlated under higher loads.
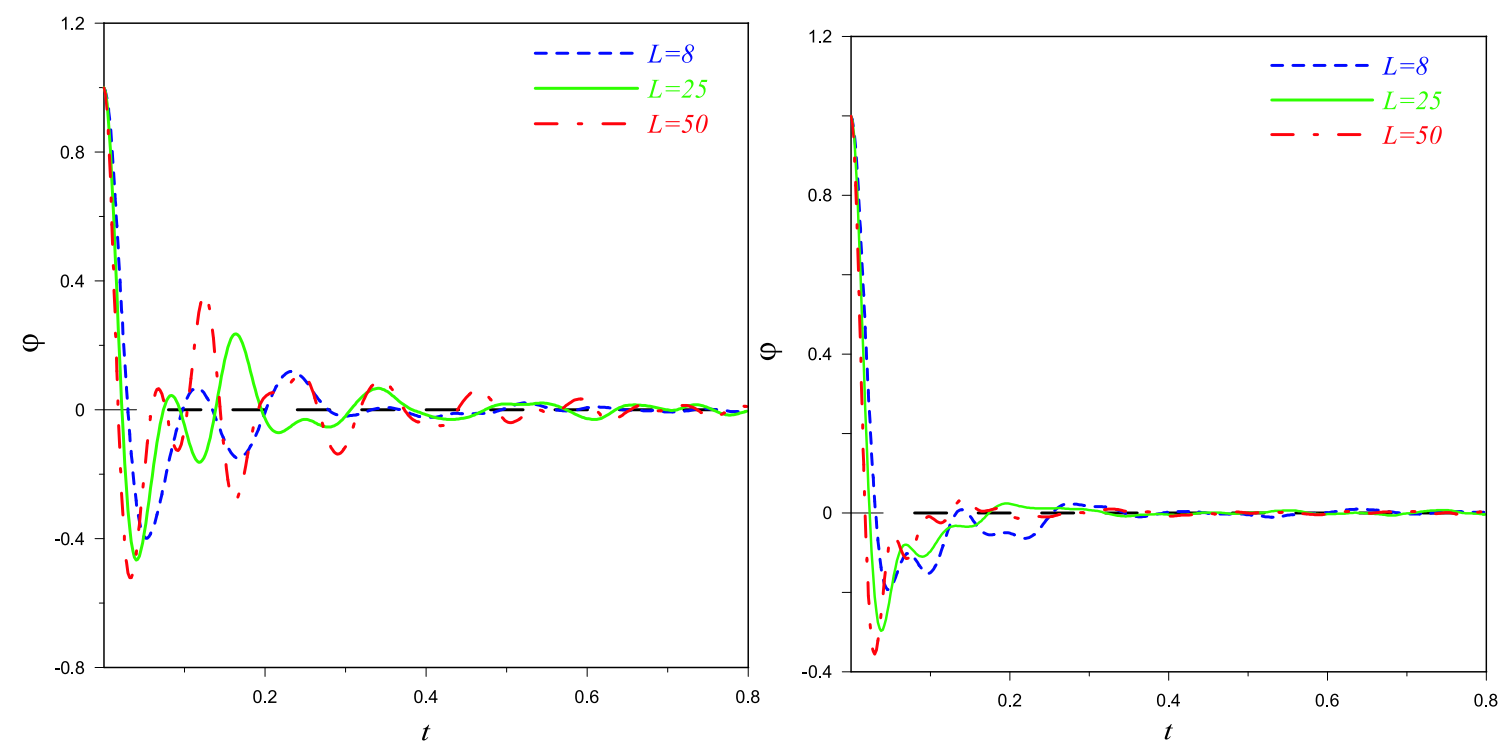

Figure 3. Time dependencies of the velocity autocorrelation function (8) for different loads and films with thickness of one (left) and two (right) molecular diameters.

A gradual decrease of the diffusion constant with the external load increase shown in figure 4 and figure 5 indicates the transition of the water film to a solidlike state. The feature of this state is the ordering of molecules which can be vertical (in the form of quasidiscrete layers) and horizontal (i. e., in-plane). Analytically the former manifests itself in the density oscillations with distance normal to the boundaries and the measure of the latter is the Debye-Waller factor. In this work, the mentioned quantities have not been calculated but ordering is observed visually. Figure 6 shows typical vertical ordering of water molecules for two values of load. In-plain ordering for one-layer film is not observed for all the load and driving force values used. However, for two molecular diameters thick film in-plane ordering takes place, especially for high loads. Typical molecule configurations are shown in figure 7 . Here one can see disordered and several types of ordered configurations of molecules. The presence of shear, as a rule, promotes the occurrence of the ordered structure for lower loads as compared with the simulation runs with zero shearing force . Moreover, shear results in a more rapid ordering. Thus, for two-layer thick water film there is a shear ordering that is observed experimentally [4]. It should be noted that for low driving forces and intermediate loads, the molecular configuration is not completely defined by parameters of these forces. For example, structures shown in figure 7 for $F_{\mathrm{S}}=0.5, L=25$ and $F_{\mathrm{S}}=0, L=30$ are not absolutely reproducible and in other simulations for these values disordered configurations are 

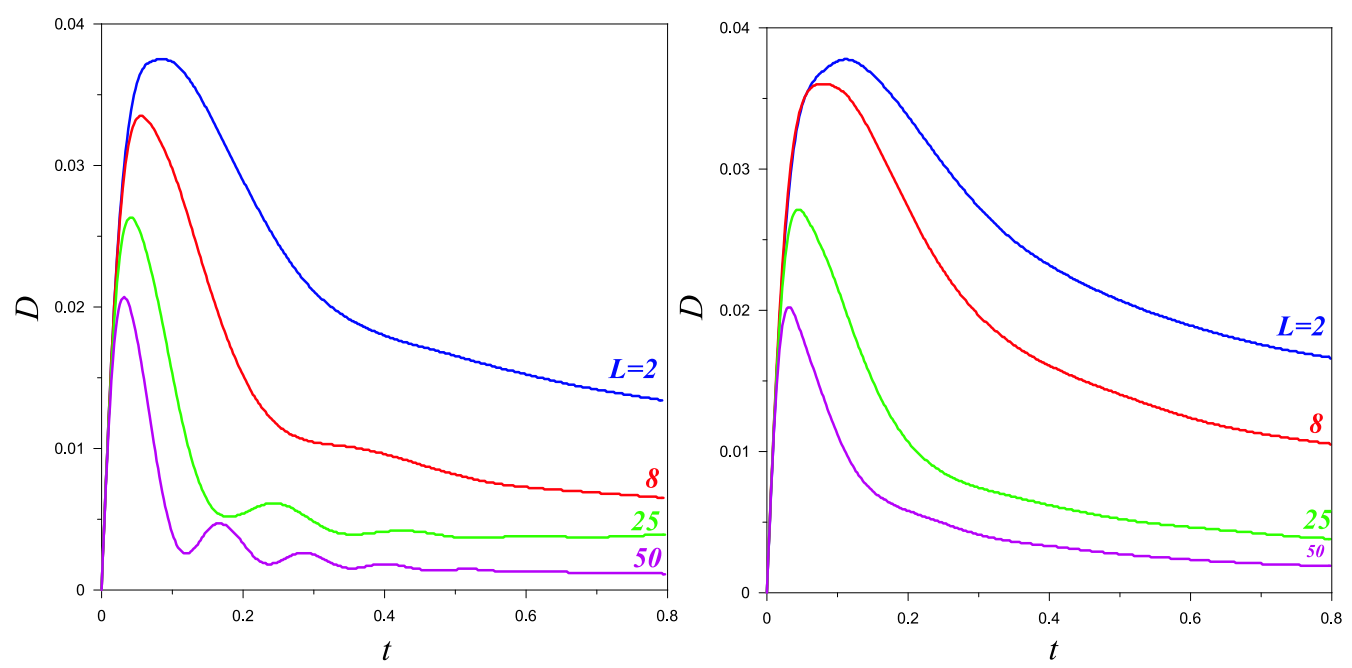

Figure 4. Time dependencies of the diffusion constant for different loads and films with thickness of one (left) and two (right) molecular diameters. Values are calculated with the Einstein expression (6).

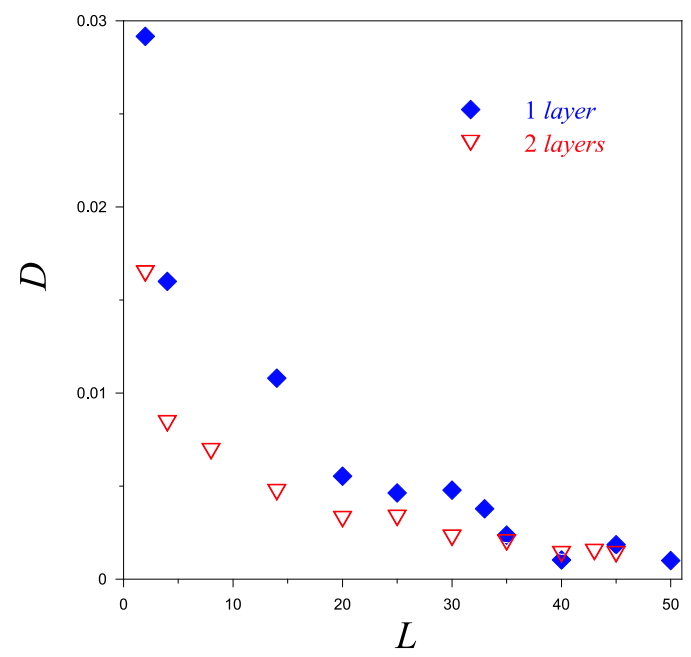

Figure 5. The diffusion constant calculated with the Green-Kubo formula (7) as function of load for films with thickness of one and two molecular diameters.

obtained. In contrast to such a behaviour for higher loads, the ordered state is always obtained as shown at the bottom right part of figure 7 .

The absence of ordering in the one-layer film can be explained having realized the model peculiarities. The behaviour of one-layer film should be similar to the one of a simple LJ liquid confined between unstructured (i.e., mathematically smooth) surface. This follows from the fact that the roughness of surfaces is negligible and in such a thin water film, its molecules can interact in the $z$ direction only with the surface atoms. Surface atoms, in turn, interact only with O sites of water molecules and only through the LJ potential. Therefore, LJ contribution dominates in the interaction of water molecules in one-layer film. For a thicker film, water molecules in adjacent layers can interact with each other and Coulomb interaction can cause the occurrence of an ordered structure. It should be noted that the observed ordering is not a real thermodynamic phase transition. Both a liquidlike and a solidlike state are not really the same as the bulk liquid or solid phases. It would probably be more correct to refer to them as static and dynamic "epistates", since they arise only in boundary films, whose properties are determined both by the confinement and the epitaxial 


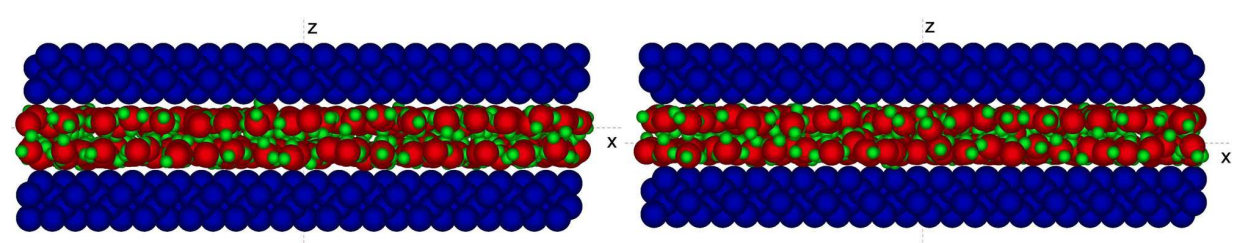

Figure 6. Layering of water molecules for values of load 8 (left) and 14 (right) and zero shear force.

interactions between the film and surface atoms [4].

Figure 8 shows time dependencies of friction force obtained for different values of shearing force. For one-layer film such a dependency is irregular with considerable fluctuations. For a thicker film, periodic spikes are observed for higher values of shearing force. Similar spikes are observed in experiments when the film is in a solidlike state and they correspond to the stick-slip friction $[4,5]$. However, a considerable difference in time values in simulations and experiments which is 10 orders of magnitude prevents from making a definite comparison of the results of simulations with the experiments (in experiments, the time values are of the order of seconds-minutes).

Time averaged friction force as a function of load is shown in figure 9. Here two main regions can be marked. The first one is linear for low $L$ and it corresponds to the first Amontons' law. The second part is horizontal and is observed for higher loads. Such dependencies can be explained using the "cobblestone model" $[3,5,16]$. According to this model, the friction force is defined by two contributions. The first one arises from the presence of internal adhesion forces between the liquid and the surface molecules. The second contribution is caused by the externally applied load. Frictional force is defined by the following expression

$$
F=S_{\mathrm{c}} A+C L,
$$

where constants $S_{\mathrm{c}}$ and $C$ are critical shear stress and the friction coefficient, $A$ is the area of contact and $L$ is the external load. Quantity $S_{\text {c }}$ depends on the adhesion interactions of the film with surfaces. The friction coefficient $C$ is related to the atomic granularity of the surfaces and depends on the size, shape, and configuration of the liquid molecules in the gap. In general, the smoother the surfaces the smaller should be the value of $C$.

Dependencies in figure 9 may be explained in the following way. Since the surfaces in simulations are smooth and water molecules are small and have a simple shape we can assume that frictional force is defined only by the adhesional contribution. Therefore, we can consider that $C \approx 0$ and in (9) there is only the first term in the right part of the equation. With the increase of load the number and strength of the adhesion bonds of water molecules with surfaces at first rapidly increase. This causes the increase of $S_{\mathrm{c}}$ and from (9) there follows an increase of the friction force with $L$ shown in figure 9. After reaching some value of the external load for the current shearing force some stationary values of the number and strength of adhesion bonds are established. With the increase of $L$ the contact area does not increase, since the model does not take into account the deformation of surfaces. So, the first term in (9) (and therefore, friction force) remains constant. The same behaviour exhibits the shear stress $S=F / A$ that is widely used in experiments. Note that in experiments for simple spherical molecules similar dependencies of shear stress $S$ on load $L$ have been obtained [5]. But for real surfaces the constancy of $S$ for high $L$ is caused by a proportional increase of $F$ and $A$ due to the deformation of surfaces. In contrast, in the simulations, the constancy of $S$ is the consequence of the constancy of both $F$ and $A$.

Thus, the considered model yields the results that in many ways correspond to simple spherical molecules and reproduce the experimentally observed first Amontontons' law for water under low loads. Nevertheless, water molecules cannot be referred to as simple spherical ones. So, to obtain more realistic results, the model should be improved. For example, the elasticity, roughness, and mutual interactions of surfaces should be taken into account. These more complicated models are the subject of further investigations. 

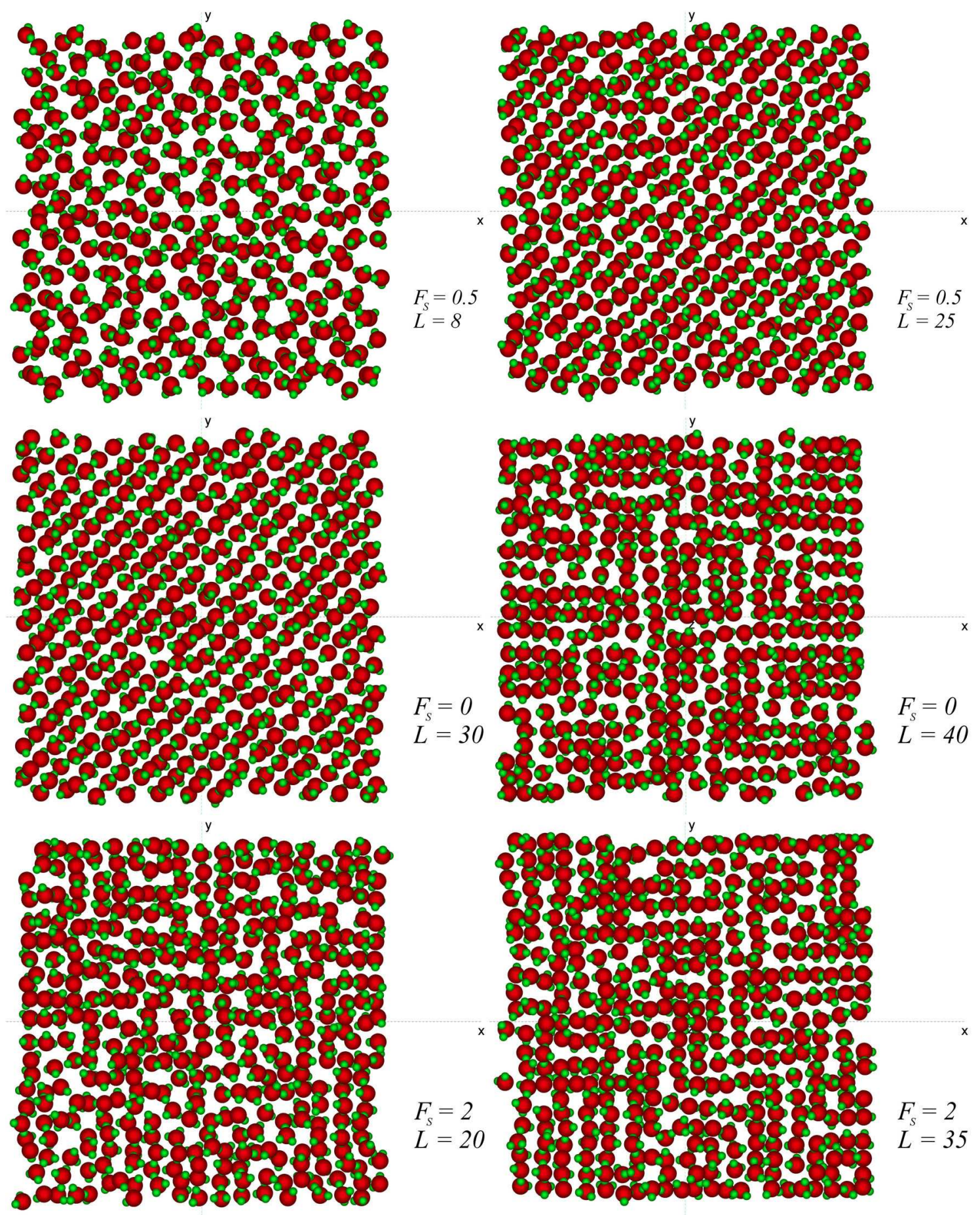

Figure 7. Configurations of molecules in $x y$ plane for different loads and shear forces at the end of corresponding simulations with the two-layer water film.

\section{Conclusion}

The main result of simulations is the transition of the ultrathin water film to a solidlike state. It manifests itself in the diffusion constant decreasing and in the ordering of molecules. One layer of molecules between flat rigid diamond plates behaves like simple LJ boundary liquid lubricant and 

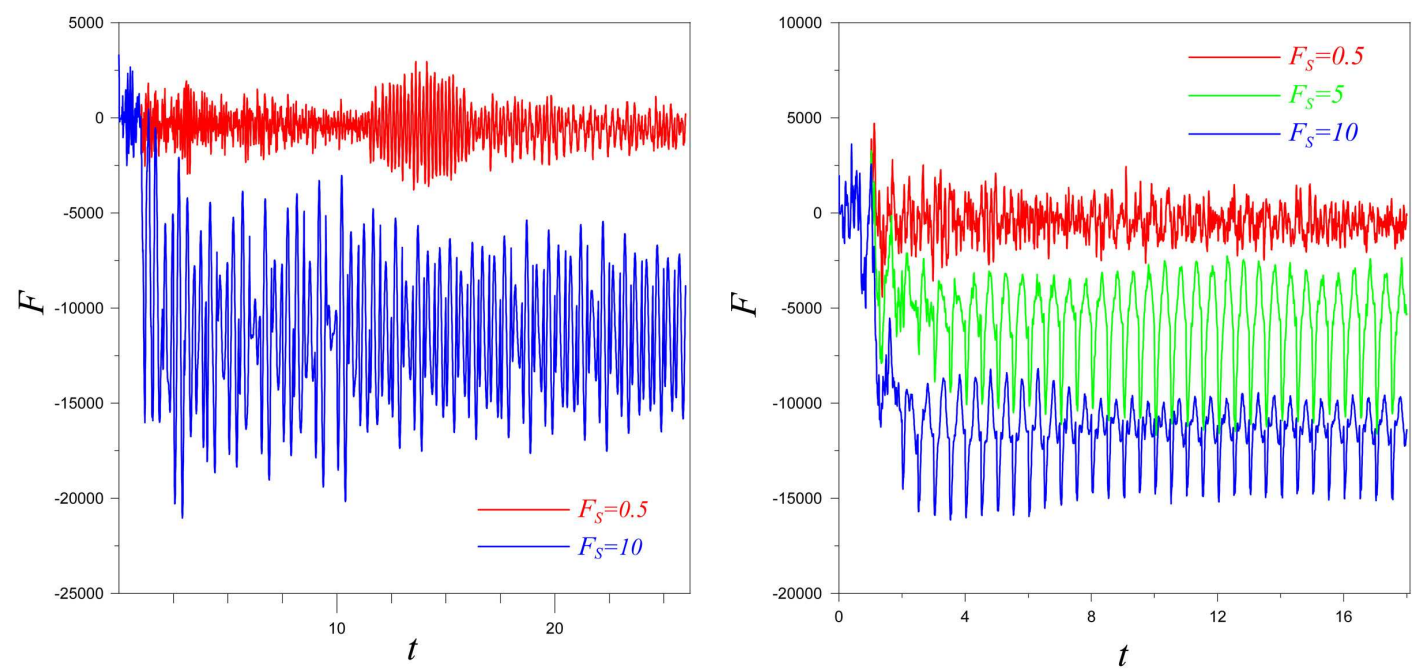

Figure 8. Time dependencies of the friction force for films with thickness of one (left) and two (right) molecular diameters and different driving forces. The value of load is 50. Negative trend is due to the fact that the friction force acts in the direction reverse to sliding.
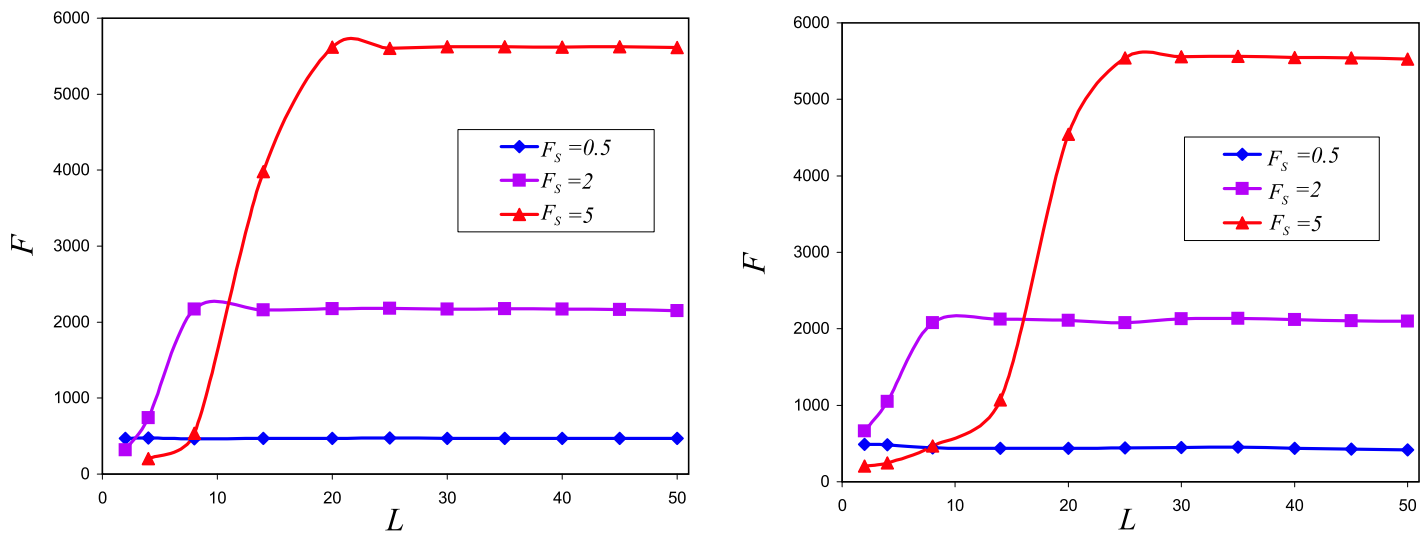

Figure 9. Time-averaged value of the friction force for films with thickness of one (left) and two (right) molecular diameters and different shearing forces as function of load.

there is no horizontal ordering in it. For a two-layer thick film, the layering and in-plane ordering are observed. The presence of a driving force causes a more rapid formation of the ordered structures and the shear ordering takes place. Such a behaviour can be explained by the peculiarities of the model, in particular by the presence of the Coulomb interactions between water molecules. Time dependencies of friction force in general reflect a solidlike state of the film and the stick-slip motion is observed for the thicker film under high shear force. However, a significant difference of time scales in simulations and experiments prevents from making a definite comparison of the corresponding results. The dependencies of time averaged values of friction force and shear stress are similar to the ones experimentally obtained for spherical molecules. They can be explained using the "cobblestone" model in the approximation of the adhesion forces domination.

\section{Acknowledgements}

We wish to express our gratitude to the Fundamental Researches State Fund (Grant No. 25.2/013) for supporting the present work and to L.S. Metlov for helpful discussions. 


\section{References}

1. Braun O.M., Naumovets A.G., Surf. Sci. Rep., 2006, 60, 79.

2. Bhushan B. Introduction - measurement techniques and applications. - In: Bhushan B. (Ed.), Nanotribology and nanomechanics. Springer, Berlin, 2005, 1-34.

3. Dedkov G.V., Physics: Uspekhi, 2000, 43, 585.

4. Yoshizawa H., Israelachvili J.N., J. Phys. Chem., 1993, 97, 11300.

5. Gee M.L., McGuiggan P.M., Israelachvili J.N., J. Chem. Phys., 1990, 93, 1895.

6. Gao J., Luedtke W.D., Landman U., J. Phys. Chem., 1997, 101, 4013.

7. Carlson J., Batista A., Phys. Rev. E., 1996, 53, 4153.

8. Popov V.L., Zh. Teh. Fiz., 2001, 71, No. 5, 100 (in Russian).

9. Aranson I.S., Tsimring L.S., Vinokur V.M., Phys. Rev. B, 2002, 65, 125402.

10. Khomenko A.V., Yushchenko O.V., Phys. Rev. E., 2003, 68, 036110.

11. Heo S.J., Sinnott S.B., Brenner D.W., Harrison J.A. Computational modeling of nanometer-scale tribology. - In: Bhushan B. (Ed.), Nanotribology and nanomechanics. Springer, Berlin, 2005, 623-691.

12. Thompson P.A., Robbins M.O., Science, 1990, 250, 792.

13. Thompson P.A., Grest G.S., Robbins M.O., Phys. Rev. Lett., 1992, 68, 3448.

14. Braun O.M., Peyrard M., Phys. Rev. E., 2001, 63, 46110.

15. Israelachvili J.N., Intermolecular and surface forces, 2nd ed. Academic Press, London, 1998.

16. Ruths M., Berman A.D., Israelachvili J.N. Surface forces and nanorheology of molecularly thin films. - In: Bhushan B. (Ed.), Nanotribology and nanomechanics. Springer, Berlin, 2005, 389-481.

17. Sansom M.S.P., Kerr I.D., Breed J., Sankararamakrishnan R., Biophys. J., 1996, 70, 693.

18. Gallo P., Ricci M.A., Rovere M., J. Chem. Phys., 2002, 116, 342.

19. Gallo P., Rovere M., J. Phys.: Condens. Matter, 2003, 15, 7625.

20. Patsahan T., Holovko M., Condens. Matter Phys., 2004, 7, 3.

21. Spohr E., Trokhymchuk A., Henderson D., J. Electroanalytical Chem., 1998, 450, 281.

22. Hua L., Huang X., Zhou R., Berne B.J., J. Phys. Chem. B, 2006, 110, 3704.

23. www.webelements.com

24. Rapaport D.C., The art of molecular dynamics simulation, 2nd ed. Cambridge University Press, Cambridge, 2004.

25. Vojutskiy S.S., The course of colloidal chemistry, 2nd ed. Chemistry, Moscow, 1975 (in Russian).

26. March N.H., Tosi M.P., Atomic dynamics in liquids, Macmillan, London, 1976. 


\section{Комп'ютерне моделювання методом молекулярної динаміки ультратонкої плівки води, стиснутої між плоскими алмазними поверхнями}

\section{О.В.Хоменко, М.В.Проданов}

Сумський державний університет, вул. Римського-Корсакова 2, 40007 Суми, Україна

Отримано 7 травня 2008 р., в остаточному вигляді - 30 вересня 2008 р.

у роботі описано комп'ютерне моделювання методом молекулярної динаміки ультратонкого шару води, стиснутого між атомарно-плоскими жорсткими алмазними поверхнями. Розглянуто плівки товщиною один і два молекулярних діаметри, для молекул води використано модель ТІР4Р. Вивчено динамічні й рівноважні характеристики системи для різних значень зовнішнього навантаження і зсувної сили. Збільшення зовнішнього тиску обумовлює перехід плівки до твердоподібного стану. Це виявляється у зменшенні коефіцієнту дифузії й утворенні молекулами рідини квазідискретних шарів. У плівці товщиною два молекулярних діаметри за високих навантажень молекули також упорядковуються паралельно до поверхонь. Отримано часові залежності сили тертя і зміни їі середнього значення із навантаженням. У цілому, поведінка моделі, що розглядається, узгоджується із експериментами для простих рідин, молекули яких мають сферичну форму.

Ключові слова: межове тертя, молекулярна динаміка, ультратонка плівка рідини

PACS: 61.20.Ja, 61.20.Ne, 61.30.Hn, 68.35.Af 